UCRL-JC-120259

PREPRINT

\title{
Resonant Soft X-Ray Fluorescence Studies of Novel Materials
}

\author{
J.A. Carlisle \\ E.L. Shirley \\ L.J. Terminello \\ E.A. Hudson \\ J.J. Jia \\ T.A. Callcott \\ F.J. Himpsel \\ D.L. Ederer \\ R.C.C. Perera
}

This paper was prepared for submittal to the Fall Meeting of the Materials Research Society

Boston, MA

November 28-December 2, 1994

February 8, 1995

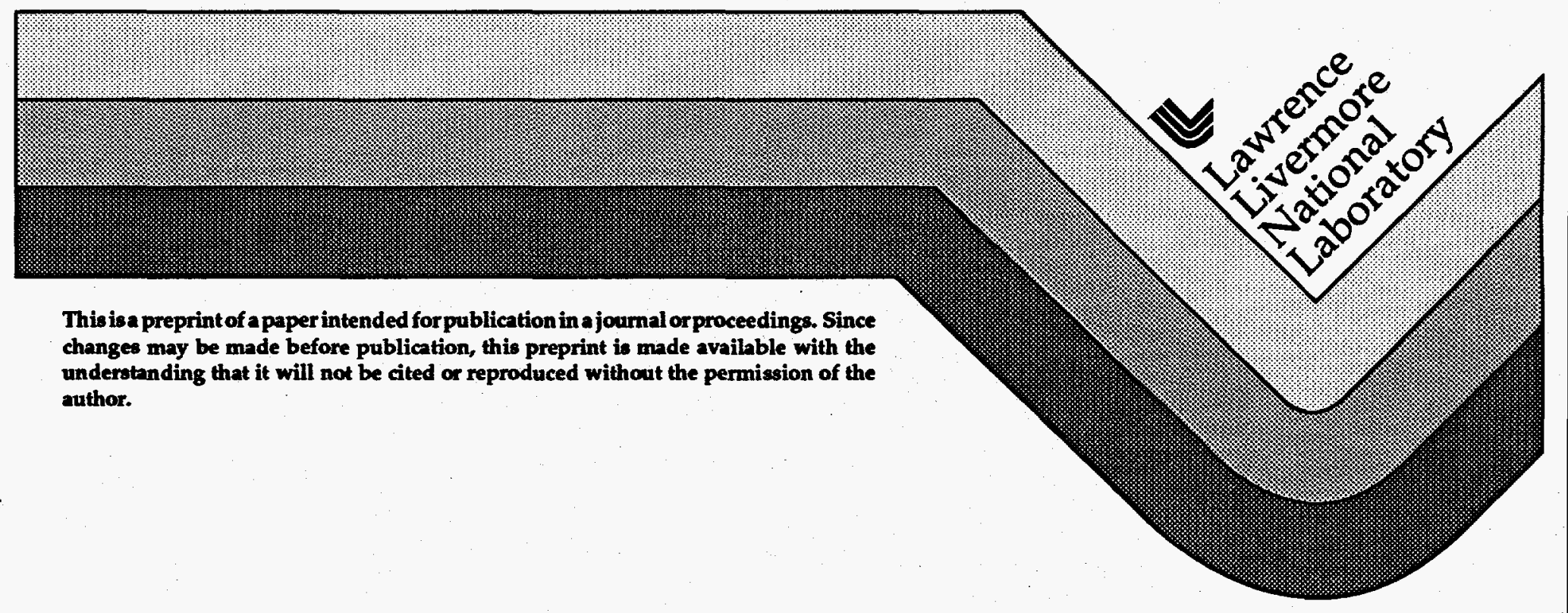




\section{DISCLAIMER}

This document was prepared as an account of work sponsored by an agency of the United States Government. Neither the United States Government nor the University of California nor any of their employees, makes any warranty, express or implied, or assumes any legal liability or responsibility for the accuracy, completeness, or usefulness of any information, apparatus, product, or process disclosed, or represents that its use would not infringe privately owned rights. Reference herein to any specific commercial product, process, or service by trade name, trademark, manufacturer, or otherwise, does not necessarily constitute or imply its endorsement, recommendation, or favoring by the United States Government or the University of California. The views and opinions of authors expressed herein do not necessarily state or reflect those of the United States Government or the University of California, and shall not be used for advertising or product endorsement purposes. 


\section{DISCLAIMER}

Portions of this document may be illegible in electronic image products. Images are produced from the best available original document. 
J. A. CARLISLE ${ }^{1}$, E.L. SHIRLEY ${ }^{2}$, L. J. TERMINELLO ${ }^{1}$, E. A. HUDSON ${ }^{1}$, J. J. JIA ${ }^{3}$, T. A. CALLCOTT $^{3}$, F. J. HIMPSEL, ${ }^{4}$ D. L. EDERER ${ }^{5}$, R.C.C. PERERA ${ }^{6}$

${ }_{1}^{1}$ Lawrence Livermore National Laboratory, Livermore, CA 94550

${ }^{2}$ National Institute of Standards and Technology, Gaithersburg, MD 20899

${ }^{3}$ University of Tennessee, Knoxville, TN 37996

${ }_{4}^{4}$ IBM Research Division, Thomas J. Watson Research Center, Yorktown Heights, NY 10598

${ }^{5}$ Tulane University, New Orleans, LA, 70118

${ }^{6}$ Lawrence Berkeley Laboratory, Berkeley, CA 74720

\section{ABSTRACT}

We are using resonant soft $\mathrm{x}$-ray fluorescence at the Advanced Light Source to probe the electronic and geometric structure of novel materials. In the resonant process, a core electron is excited by a photon whose energy is near the core binding energy. In this energy regime the absorption and emission processes are coupled, and this coupling manifests itself in several ways. In boron nitride (BN), the resonant emission spectra reflect the influence of a "spectator" electron in an unoccupied excitonic state. The resonant emission can be used to distinguish between the various bulk phases of $\mathrm{BN}$, and can also be used to probe the electronic structure of a monolayer of $\mathrm{BN}$ buried in a bulk environment, where it is inaccessible to electron spectroscopies. For highly-oriented pyrolytic graphite (HOPG) a coherent absorption-emission process takes place in the resonant regime, whereby crystalline momentum is conserved between the core excited electron and the valence hole which remains after emission

\section{INTRODUCTION}

Soft x-ray fluorescence (SXF) spectroscopy using synchrotron radiation offers several advantages over surface sensitive spectroscopies for probing the electronic structure of complex multi-elemental materials [1]. Due to the long mean free path of photons in solids $(\sim 1000 \AA)$, SXF is a bulk-sensitive probe. Also, since core levels are involved in absorption and emission, SXF is both element- and angular-momentum-selective. SXF measures the local partial density of states (DOS) projected onto each constituent element of the material. The chief limitation of SXF has been the low fluorescence yield for photon emission, particularly for light elements. However, third generation light sources, such as the Advanced Light Source (ALS), offer the high brightness that makes high-resolution SXF experiments practical. In the following we utilize this high brightness to demonstrate the capability of SXF for detecting the electronic structure of monolayer quantities at buried interfaces and to probe the band structure of a polycrystalline sample.

In SXF, a valence emission spectrum results from transitions from valence band states to the core hole produced by the incident photons. In the non-resonant energy regime, the excitation energy is far above the core binding energy, and the absorption and emission events are uncoupled. The fluorescence spectrum resembles emission spectra acquired using energetic electrons, and is insensitive to the incident photon's energy. In the resonant excitation energy regime, core electrons are excited by photons to unoccupied states just above the Fermi level $\left(E_{F}\right)$. The absorption and emission events are coupled, and this coupling manifests itself in several ways, depending in part on the localization of the empty electronic states in the material. 
In boron nitride, the valence emission spectrum at resonance is shifted, and reflects the influence of an electron excited to a localized, excitonic state (a $\pi^{*}$ state) [2]. The presence of the localized electron screens the valence electrons, resulting in a shift of the valence emission to lower photon energies. Emission is also observed from (normally unoccupied) excitonic (quasibound) states above $\mathrm{E}_{\mathrm{F}}$. Resonant $\mathrm{SXF}$ experiments involving delocalized final states in graphite [3], Si [4], and Diamond [5], have found dispersive effects and intensity modulations in the valence emission spectrum as the incident photon energy is varied.

\section{RESONANT FLUORESCENCE STUDIES BURIED INTERFACES}

Fig. 1 illustrates the use of the resonant SXF technique for probing the structure of different bulk phases of BN. In Fig. 1a is shown the boron 1s fluorescence spectra acquired from cubic $(\mathrm{cBN})$ and hexagonal $(\mathrm{hBN})$ boron nitride. These spectra were obtained using a near-resonant excitation energy of $206 \mathrm{eV}$. Although the valence band emission region is slightly different for $\mathrm{cBN}$ and $\mathrm{hBN}$, by far the most striking difference is the sharp feature at $193 \mathrm{eV}$ from $\mathrm{hBN}$. Its presence in $\mathrm{hBN}$ and absence in $\mathrm{cBN}$ is easily understood in light of recent photoabsorption data
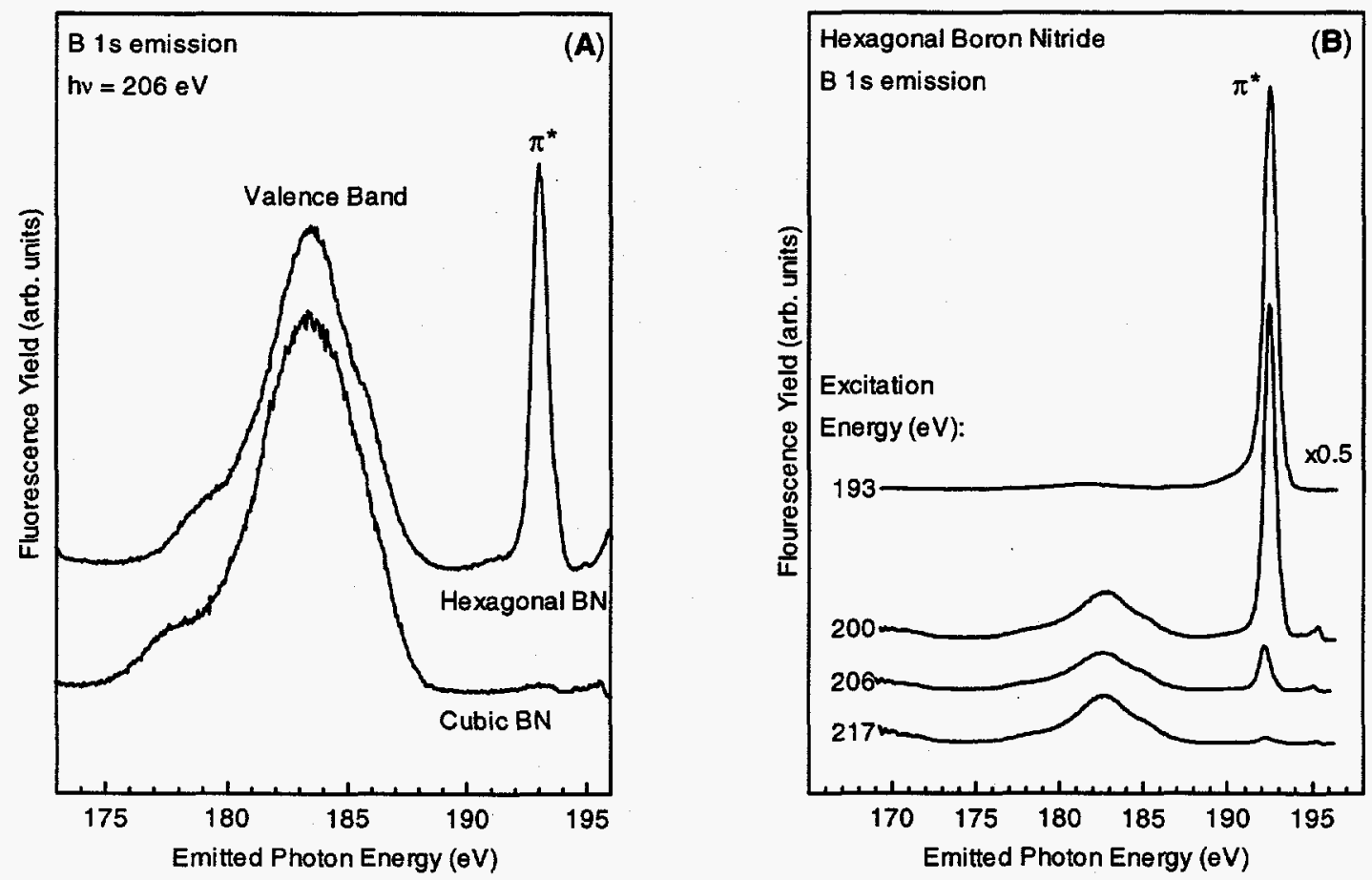

Fig. 1: (a) Soft $x$-ray fluorescence spectra measured from hexagonal and cubic BN. Hexagonal BN is $\pi$ bonded. Emission from quasi-bound $\pi^{*}$ states at $193 \mathrm{eV}$ may be viewed in the resonant fluorescence spectrum when in excitation energy is tuned near the $\pi^{*}$ resonance energy. Cubic BN is $\sigma$ bonded (e.g. diamond). There are no empty states near the Fermi level in this system, so no emission from quasibound states above the Fermi level are detected, as expected. The presence of the quasi-bound feature is thus indicative of the geometric and electronic structure. (b) Resonant fluorescence spectra from hexagonal BN. As the excitation energy is tuned to the $\pi^{*}$ resonance, the emission from the quasibound state is greatly enhanced

on these systems [6]. The peak at $193 \mathrm{eV}$ results from resonant emission from an excitonic state 
which corresponds to the (normally unoccupied) $\pi^{*}$ anti-bonding state present when the boron in these systems is $\pi$ bonded. Hexagonal BN is $\mathrm{sp}^{2}$-bonded, and thus exhibits a $\pi^{*}$ resonance feature. Since $\mathrm{cBN}$ has the diamond structure and is $\mathrm{sp}^{3}$ bonded, there are no empty quasibound states in the region just above $E_{F}$ from which resonant emission can take place. Thus, the presence of this $\pi^{*}$ resonance feature is indicative of both the geometric and electronic structure.

Fig. 1b shows that, in addition to using this feature as a marker for $\pi$-bonded boron, the resonance feature can also be used to detect very small quantities of boron in a material. As the incident photon energy is tuned to the resonant energy of $193 \mathrm{eV}$, the magnitude of the resonance increases dramatically. It's peak intensity is nearly 30,000 times more than the peak valence emission intensity using this excitation energy. The resonant peak's intensity would be even higher were it not for self-absorption effects, which reduce it's intensity relative to the valence band emission.

The potential of using resonant SXF for detecting small quantities of $\pi$-bonded boron based on the results above lead us to construct two buried layer samples, one of which is shown in Fig 2. These buried BN layer samples were synthesized by magnetron sputter-depositing $3 \AA$ of BN onto either a clean $\mathrm{Si}(100)$ substrate or an amorphous $50 \AA$ thick carbon buffer layer on Si that were encapsulated with $50 \AA$ of amorphous carbon. The samples studied here consist of a $3 \AA$ layer of $\mathrm{BN}$, and the combination of the very strong resonant feature and the high brightness of the ALS enabled us to detect the buried heterojunction in less than five minutes of data accumulation and to collect statistically significant spectra in less than thirty minutes. It should be noted that, as synthesized, these samples are inaccessible to electron-based spectroscopies. Although the depth distribution of boron (as well as the other elements present) may be obtained via transmission electron microscopy (TEM) or Rutherford backscattering spectroscopy (RBS), no technique to our knowledge allows the concurrent extraction of element specific electronic information from samples such as these.

Resonant SXF spectra acquired from the buried monolayer samples are shown in Fig. 3. SXF data obtained from a $\mathrm{hBN}$ sample under identical conditions are also shown for comparison. The spectra shown in Fig. 3 have been normalized so that the effects of sample position and different incident photon fluxes have been eliminated. As can be seen in Fig. 3, the resonant fluorescence signal from both the $\mathrm{C} / \mathrm{BN} / \mathrm{C}$ and $\mathrm{C} / \mathrm{BN} / \mathrm{Si}$ samples is readily detectable. It is quite clear, however, that the strength of the $\pi^{*}$ related signal is much greater for the C/BN/C system than in the C/BN/Si system. This implies that for equal quantities of $\mathrm{BN}$ in each sample, the $\mathrm{BN}$ monolayer present in the $\mathrm{C} / \mathrm{BN} / \mathrm{C}$ sample is much more $\pi$-bonded than the $\mathrm{BN}$ in the $\mathrm{C} / \mathrm{BN} / \mathrm{Si}$ sample. Thus, the electronic structure of the buried boron in each of these systems is significantly different. Evidently, the local order of the buried BN layer is hexgonal-like for the $\mathrm{C} / \mathrm{BN} / \mathrm{C}$ system as opposed to cubic-like for the C/BN/Si sample.

It is clear from Fig. 3 that the detection limit for this technique in the present experiment is much less than one monolayer. The peak to backgound ratio for the $\mathrm{C} / \mathrm{BN} / \mathrm{C}$ interface is $\sim 180$, so for this system and apparatus the detection limit is about 0.006 monolayers. In the present experiment a high-resolution $(0.3 \mathrm{eV})$ detector was used, but clearly this is not required to detect the resonant peak. If instead a germanium detector was used, which has a much higher quantum efficiency and several orders larger detection angle, the detection limit could theoretically be much, much lower. 


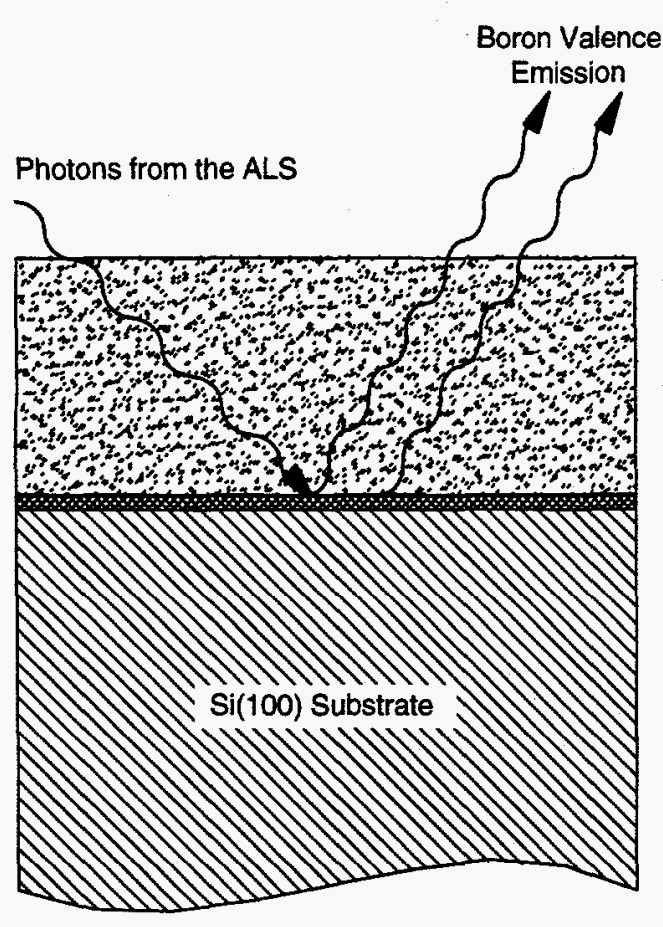

Fig. 2. One of the buried monolayer samples studied in this work. Both samples were created via magnetron sputtering a $3 \AA$ layer of $B N$ onto either a clean $\mathrm{Si}(100)$ substrate or an amorphous carbon buffer layer.

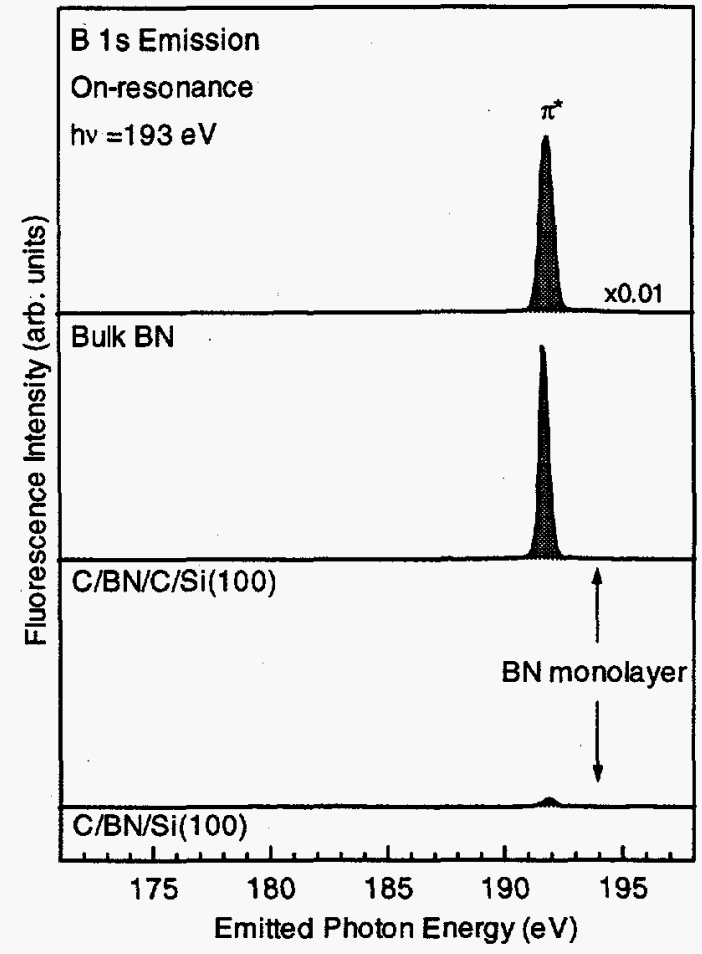

Fig. 3. Resonant fluorescence spectra from the buried monolayer samples in Fig. 2 and from a bulk hexagonal sample. The $\pi^{*}$ resonance feature is much more intense in the $\mathrm{C} / \mathrm{BN} / \mathrm{C}$ sample as opposed to the C/BN/Si sample, which indicates that the $B N$ monolayer in C/BN/C is more $\pi$-bonded than in C/BN/Si.

\section{BAND MAPPING USING PHOTONS: COHERENT FLUORESCENCE IN GRAPHITE}

We will now show that the coupling between absorption and emission in delocalized materials allows one to probe band structure in a unique way. Resonant versus off-resonant fluorescence spectra that were measured from highly oriented pyrolytic graphite (HOPG) at the ALS are shown in Fig. 4a. The take-off angle for these data was $35^{\circ}$, and the width of the excitation energy was approximately $0.3 \mathrm{eV}$. The uppermost spectrum is characteristic for the nonresonant case, far above threshold $\left(\mathrm{h} v_{\text {in }}=400 \mathrm{eV}\right)$. Peaks in the off-resonant spectrum derive primarily from flat regions in the band structure (such as around critical points), which are regions having a high DOS. The resonant spectra are shown below the non-resonant spectrum. Most of these spectra have a drastically different line shape compared to the non-resonant one. Instead of intensity fluctuations observed in other delocalized materials, most of the emission peaks appear to disperse [5,7]. Their emitted photon energies $\left(h v_{\text {out }}\right)$ change as $h v_{\text {in }}$ is varied from $284 \mathrm{eV}$ to $287 \mathrm{eV}$. These dispersive peaks are highlighted by the dashed lines and are numbered 1-7 in Fig. 4a. The changes in the emission spectrum are most dramatic when $\mathrm{hv}$ in is varied by just $0.5 \mathrm{eV}$, from $284.5 \mathrm{eV}$ to $285 \mathrm{eV}$. The intensities of features 3 and 4 abruptly increase, while peaks 2 and 3 diminish substantially. Such large changes in the spectrum due to 
such small changes in $\mathrm{hv}_{\text {in }}$ cannot be accounted for solely by simple intensity modulations, which have been explained in terms of sweeping through critical points in the Brillouin zone [5,7].

The $h v_{\text {in }}$-dependent emission features are the result of transitions from states with a well defined crystal momentum. The upper panel of Fig. 4a shows the band structure of graphite along the high symmetry directions as derived from a tight-binding parametrization of quasiparticle calculations [8]. The $\sigma$ and $\pi$ molecular orbitals give rise to the bands labeled in Fig. 4a, and correspond to the different types of bonding ( $\sigma$ or $\pi$ bonding) which arise between carbon atoms in the graphite sheets. In this figure the band structure has been rotated and the binding energy axis aligned to the lower panel's photon energy axis.

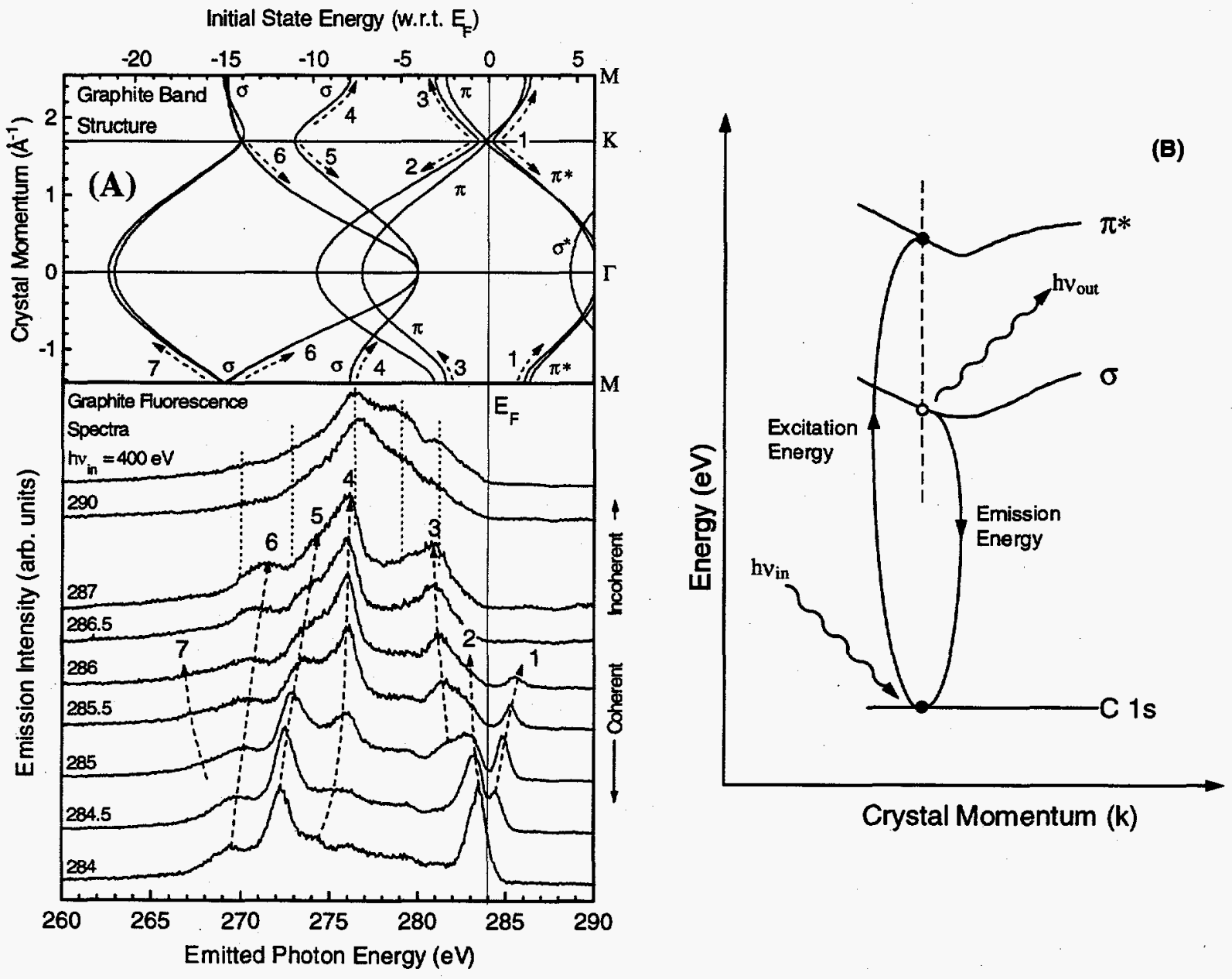

Fig. 4 (a) Resonant and non-resonant fluorescence spectra from highly-oriented pyrolytic graphite at incident energies $h v_{i n}$. The upper panel displays the graphite band structure with the energy axis matched to the photon energy axis of the fluorescence data. The dispersive features labeled 1-7 in the resonant spectra are associated with the portions 1-7 of the graphite band structure indicated by dashed lines and arrows in the upper panel. (b) Schematic representation of coherent fluorescence. After the incident photon excites the core electron to the empty $\pi^{*}$ band, only emission from occupied states with the same crystal momentum (vertical line) is allowed

Fig. 4b shows schematically the coherent absorption-emission process. Control over $\mathrm{hv}_{\text {in }}$ allows one to select the crystal momentum of the photoelectron's final state in the conduction band. 
Thus the allowed transitions from occupied states to fill the core hole lie on a vertical line through the band structure having the same crystal momentum as the electron in the $\pi^{*}$ conduction band. One may predict the dispersive behavior of the emission peaks graphically in Fig. $4 \mathrm{~b}$ by constructing sets of vertical lines whose $\mathbf{k}$-values are determined by which $\mathbf{k}$-point in the $\pi^{*}$ band the core electron is excited to by $h v_{i n}$. The allowed transitions (along the high symmetry directions only) are determined by where the lines intersect the bands. The result of such an analysis leads in a straightforward way to the association of different portions of the graphite band structure in the upper panel of Fig. 4a, which are labeled 1-7, with the emission features labeled 1-7 in the lower panel. Clearly, there is a correlation between the dispersive emission features and the $\sigma$ and $\pi$ bands of the HOPG band structure.

\section{SUMMARY}

To summarize, we have demonstrated the value of SXF as a non-destructive technique to study the electronic bonding of buried interfaces and the momentum-resolved electronic structure of polycrystalline samples. The electronic information gathered from the systems studied in this .work is inaccessible to traditional surface sensitive techniques. To our knowledge the elementspecific electronic structure of a buried heterointerface has not been realized previously. Although we have used resonant SXF to probe the electronic properties of a Boron-containing monolayer, the technique should be applicable to other systems with localized antibonding states. The local momentum-resolved electronic configuration on each constituent element of a more complex (possibly insolating) material may be characterized with this technique as well.

Acknowledgment: This work was supported by the Division of Materials Science, Office of Basic Energy Sciences, and performed under the auspices of the U.S. Department of Energy by Lawrence Livermore National Laboratory under contract No. W-7405-ENG-48, by National Science Foundation Grant No. DMR-9017996 and DMR-9017997, by a Science Alliance Center for Excellence Grant from the University of Tennessee, and by the U.S. Department of Energy (DOE) Contract No. DE-AC05$840 R 21400$ with Oak Ridge National Laboratory. This work was performed at the Advanced Light Source, which is also supported by the Office of Basic Energy Sciences, U.S Department of Energy, under contract No. DE-AC03-76SF00098. .

\section{REFERENCES}

[1] D.L. Ederer, T.A. Callcott, and R.C.C. Perera, Synchrotron Radiation News 7, 29 (1994).

[2] J-E. Rubensson, D. Mueller, R. Shuker, D.L. Ederer, C.H. Zhang, J. Jia, and T.A. Callcott, Phys. Rev. Lett. 64, 1047 (1990).

[3] J.A. Carlisle, E.L. Shirley, E.A. Hudson, L.J. Terminello, T.A. Callcott, J.J. Jia, D.L. Ederer, R.C.C. Perera, and F.J. Himpsel, Phys. Rev. Lett. (in press).

[4] K.E. Miyano, D.L. Ederer, T.A. Callcott, W.L. O'Brien, J.J. Jia, L. Zhou, Q.-Y. Dong, Y. Ma, J.C.Woicik, and D.R. Mueller, Phys. Rev. B 48, 1918 (1993).

[5] Y.Ma, N. Wasshahl, P. Skytt, J. Guo, J. Nørdgren, P.D. Johnson, J-E. Rubensson, T. Boske, W. Eberhardt, and S. D. Kevan, Phys. Rev. Lett. 69, 2598 (1993).

[6] A. Chaiken, L.J. Terminello, J. Wong, G.L. Doll, and C.A. Taylor II, Appl. Phys. Lett. 63, 2112 (1993).

[7] P.D. Johnson, Y. Ma, Phys. Rev. B 49, 5024 (1994).

[8] X. Zhu and S. G. Louie, unpublished; see also I.T. McGovern, W. Eberhardt, E.W. Plummer, and J.E. Fischer, Physica 99B, 415 (1980), and R.F. Willis, B. Feuerbacker, and B. Fitton, Phys. Rev. B 4, 2441 (1971). 